INFORMATION STUDIES DAYS 2020

\title{
Health literacy supports active aging
}

\section{Johanna Eronen}

Gerontologian tutkimuskeskus, liikuntatieteellinen tiedekunta, Jyväskylän yliopisto johanna.eronen@jyu.fi

https://orcid.org/0000-0002-5641-9156

Leena Paakkari

Erja Portegijs

Milla Saajanaho

Taina Rantanen

Keywords: gerontology, health promotion, comorbidity

This article is licensed under the terms of the CC BY-NC-SA 4.0 -license

Persistent identifier: https://doi.org/10.23978/inf.98618 


\section{Background}

Discussion around active aging has centered on societal values, norms and benefits related to productivity, with older people regarded as a social and economic resource, and indicators of active aging emphasizing the importance of employment, increased life expectancy and independence (Foster \& Walker, 2015). Moreover, the measures of active aging have been conducted at the societal level (Zaidi et al, 2017). From the individual perspective, the will to perform self-selected meaningful activities has been identified as a key factor in active aging (Rantanen et al, 2018a; Stephens et al, 2015). To explore active aging at the individual level, we define it as "the striving for elements of wellbeing through activities relating to a person's goals, functional capacities and opportunities" (Rantanen et al. 2018a). A new measurement of active aging (the University of Jyväskylä Active Aging Scale, UJACAS) is based on the viewpoint of individuals and the resources they need to pursue what is personally important for them, and it acknowledges individuals' participation in regular every-day activities. Using this measurement, the aim of this study was to examine the association between active aging and health literacy among older persons with varying numbers of chronic conditions.

\section{Methods}

The participants were 75, 80 and 85-year-old independently living men and women ( $n=949)$. Active aging was measured with the University of Jyväskylä Active Aging Scale (UJACAS) (Rantanen et al. 2018a), and health literacy with the Finnish translation of the short version of the European Health Literacy Survey (HLS-EU-Q16) (Sørensen et al, 2013; Eronen et al, 2019). Physiciandiagnosed chronic conditions were self-reported and participants' gender and age drawn from the Finnish National Population Register (Rantanen et al 2018b). Length of education, living situation (alone or with someone), depressive symptoms, cognitive capacity and physical performance were considered as potential confounders. Linear regression analyses and general linear model were used to estimate the associations between active aging, health literacy and number of chronic conditions. 


\section{Results}

Nearly half of the participants (47\%) were $75,33 \%$ were 80 and $20 \%$ were 85 years old. The proportion of women was $56.3 \%$. Of all participants, $53 \%$ had sufficient, $35 \%$ problematic and $12 \%$ inadequate health literacy. The 75 -yearolds showed the highest proportion of participants with sufficient health literacy (60.5\%) and the 85-year-olds the highest proportion of those with inadequate health literacy (18.8\%). The mean number of chronic conditions was 3.4. Linear regression analyses, adjusted for all confounders, showed that health literacy $(\beta$ o.18, $\mathrm{p}<0.001)$ and chronic conditions $(\beta-0.06, \mathrm{p}=0.021)$, were associated with active aging. When the number of chronic conditions was divided into categories (o-1, 2, 3, 4, 5 and 6 or more conditions), in each category the participants with sufficient health literacy had significantly higher active aging scores than those with problematic or inadequate health literacy.

\section{Conclusions}

Our findings suggest that higher levels of health literacy enable older persons to maintain higher levels of active aging, including those with multiple chronic conditions. It seems that high health literacy buffers against the negative consequences of chronic conditions, and it may also capture an individual's resources for coping with illnesses, such as dealing with medications and visiting health care for regular check-ups. Moreover, better self-care skills of individuals with good health literacy may also underlie the more positive perception of active aging (Osborn et al, 2011). In addition, it is possible that persons with higher health literacy also possess other resources that enable them to find ways to continue engaging in activities meaningful to them irrespective of chronic conditions (Siltanen et al, 2019; Tourunen et al, 2019). An older person with chronic conditions is often a health care client, and to respond to their individual needs, health care workers need competence to identify patients and clients whose health literacy skills are below optimal, and ability to adapt their communication according to the client's level of health literacy (van der Heide et al, 2018). 


\section{References}

Eronen, J., Paakkari, L., Portegijs, E., Saajanaho, M., \& Rantanen, T. (2019). Assessment of health literacy among older Finns. Aging Clinical and Experimental Research, 31(4), 549-556. https://doi.org/10.1007/s40520-018-1104-9

Foster, L., \& Walker, A. (2015). Active and Successful Aging: A European Policy Perspective. The Gerontologist, 55(1), 83-90. https://doi.org/10.1093/geront/gnue28

Osborn, C.Y., Paasche-Orlow, M., Bailey, S.C., \& Wolf, M.S. (2011). The mechanisms linking health literacy to behavior and health status. American Journal of Health Behavior, 35(1), 118-128. https://doi.org/10.5993/ajhb.35.1.11

Rantanen, T. (a), Portegijs, E., Kokko, K., Rantakokko, M., Törmäkangas, T., \& Saajanaho, M. (2018). Developing an assessment method of active aging: University of Jyvaskyla Active Aging Scale. Journal of Aging and Health, 31(6), 1002-1024. https://doi .org/10.1177/0898264317750449

Rantanen, T. (b), Saajanaho, M., Karavirta, L., Siltanen, S., Rantakokko, M., Viljanen, A., Rantalainen, T., Pynnönen, K., Karvonen, A., Lisko, I., Palmberg, L., Eronen, J., Palonen, E.-M., Hinrichs, T., Kauppinen, M., Kokko, K., \& Portegijs, E. (2018). Active aging - resilience and external support as modifiers of the disablement outcome: AGNES cohort study protocol. BMC Public Health, 18(1). https : //doi.org/10.1186/s12889-018-5487-5

Siltanen, S., Portegijs, E., Saajanaho, M., Poranen-Clark, T., Viljanen, A., Rantakokko, M., \& Rantanen, T. (2018). The combined effect of lower-extremity function and cognitive performance on perceived walking ability among older people: a 2-year follow-up study. Journals of Gerontology: Series A Biological and Medical Sciences, 73(11), 1568-1573. https://doi. org/10.1093/gerona/gly103

Sørensen, K., van den Broucke, S., Pelikan, J., Fullam, J., Doyle, G., Slonska, S., Kondilis, B., Stoffels, V., Osborne, R.H., \& Brand, H. (2013). Measuring health literacy in populations: illuminating the design and development process of the European Health Literacy Survey Questionnaire (HLS-EU-Q). BMC Public Health, 13(1). https://doi .org/10.1186/1471-2458-13-948

Stephens, C., Breheny, M., \& Mansvelt, J. (2015). Healthy aging from the perspective of older people: A capability approach to resilience. Psychology and Health, 3o(6), 715-731. https:// doi.org/10.1080/08870446.2014.904862

Tourunen, A., Siltanen, S., Portegijs, E., Eronen, J., Rantanen, T., \& Saajanaho, M. (2019). Assimilative and Accommodative Coping and Older People's Leisure Activities. Journal of Aging and Health, 089826431985200. https://doi.org/10.1177/0898264319852002

Zaidi, A., Gasior, K., Zolyomi, A.S., Rodrigues, R., \& Marin, B. (2017). Measuring active and healthy aging in Europe. Journal of European Social Policy, 27(2), 138-157. https://doi. org/10.1177\%2F0958928716676550 